\title{
EFFECT OF INFECTION BY PLASMODIUM FALCIPARUM ON THE MELANIZATION IMMUNE RESPONSE OF ANOPHELES GAMBIAE
}

\begin{abstract}
LOUIS LAMBRECHTS,* ISABELLE MORLAIS, PARFAIT H. AWONO-AMBENE, ANNA COHUET, FRÉDÉRIC SIMARD, JEAN-CLAUDE JACQUES, CATHERINE BOURGOUIN, AND JACOB C. KOELLA

Laboratoire de Parasitologie Evolutive, Centre National de la Recherche Scientifique-Université Pierre et Marie Curie, Paris, France; Centre de Production et d'Infection des Anophèles, Institut Pasteur, Paris, France; Laboratoire de Recherche sur le Paludisme, Institut de Recherche pour le Développement-Organisation de Coordination pour la Lutte contre les Endémies en Afrique Centrale, Yaoundé, Cameroon; Unité de Caractérisation et Contrôle des Populations de Vecteurs, Institut de Recherche pour le Développement, Montpellier, France; Unité de Biologie et Génétique du Paludisme, Institut Pasteur, Paris, France
\end{abstract}

\begin{abstract}
Melanization is an immune response of mosquitoes that could potentially limit Plasmodium development. That mosquitoes rarely melanize Plasmodium falciparum in natural populations might result from immuno-suppression by the parasite, as has been observed in Aedes aegypti mosquitoes infected by Plasmodium gallinaceum. We tested this possibility in Anopheles gambiae mosquitoes infected by $P$. falciparum by comparing the ability to melanize a Sephadex bead of infected mosquitoes, of mosquitoes that had fed on infectious blood without becoming infected, and of control mosquitoes fed on uninfected blood. Rather than being immuno-suppressed, infected mosquitoes tended to have a stronger melanization response than mosquitoes in which the infection failed and than control mosquitoes, possibly because of immune activation after previous exposure to invading parasites. This finding suggests that $P$. falciparum relies on immune evasion rather than immuno-suppression to avoid being melanized and confirms that natural malaria transmission systems differ from laboratory models of mosquito-Plasmodium interactions.
\end{abstract}

\section{INTRODUCTION}

Successful transmission of the human malaria parasite Plasmodium falciparum by anopheline mosquitoes is determined by complex interactions between the parasite and its vector. Understanding these interactions is helpful to identify weaknesses that could be manipulated to reduce malaria transmission. ${ }^{1}$ Many vector-parasite interactions engage the mosquito's innate immune system, a powerful system of defense. ${ }^{2}$ Immune activation of mosquitoes after a $P$. falciparumcontaining blood meal ${ }^{3}$ is believed to limit the development of malaria parasites in a variety of ways. ${ }^{4}$ However, although the number of invading parasites is generally reduced by several orders of magnitude, a small number of parasites are often able to complete their development in the mosquito. ${ }^{5,6}$ How do these parasites overcome the mosquito's immune response?

In an attempt to study this question, we focused on one of the major components of the immune system of mosquitoes: the melanization response. ${ }^{7}$ This immune response received considerable attention as a potential resistance mechanism against Plasmodium after the artificial selection of a refractory line of the mosquito Anopheles gambiae that melanizes the parasite at the late ookinete stage. ${ }^{8,9}$ This attention was revived by the recent identification of several mosquito genes that are to some extent associated with the melanization of malaria parasites in $A$. gambiae. ${ }^{10-13}$

However, although it is clear that melanization can lead to resistance against malaria infection at least after a selection treatment in the laboratory, ${ }^{8,9}$ it is very rare to find mosquitoes in natural populations that have melanized their parasites. For example, in one study of field-caught $A$. gambiae in Tanzania, $<0.5 \%$ of infected mosquitoes harbored melanized oocysts of $P$. falciparum. ${ }^{14}$ This is all the more surprising because, in the same study, $\sim 90 \%$ of the mosquitoes readily

* Address correspondence to Louis Lambrechts, Department of Entomology, University of California, One Shields Ave., Davis, CA 95616. E-mail: 1lambrechts@ucdavis.edu melanized negatively charged Sephadex beads inoculated into the insect's hemolymph. ${ }^{14}$

Thus, the lack of $P$. falciparum melanization by wild $A$. gambiae does not seem to result from a general lack of immuno-competence of the mosquitoes but rather from a mechanism allowing the parasite to bypass this immune response. One possible explanation is that the parasite is able to hide from the immune response (i.e. to evade it). For example, mosquito-derived proteins incorporated into the oocyst capsules of the avian malaria parasite Plasmodium gallinaceum might mask developing oocysts from the mosquito's immune system. ${ }^{15}$ The observation that the absence of two mosquito C-type lectins, CTL4 and CTLMA2, results in massive melanization of parasites in a susceptible line of $\mathrm{A}$. gambiae supports the idea that the parasite can use some molecules produced by the mosquito to evade the immune response. ${ }^{10}$ Alternatively, the parasite might be able to suppress or at least reduce the effectiveness of the melanization response. Indeed, host immuno-suppression by pathogens is often observed, for example, inhibition of immune hemocyte function by insect polydnaviruses, ${ }^{16}$ blocking of host cell phagocytosis by Yersinia pseudotuberculosis,${ }^{17,18}$ disruption of plant immune signalling by a fungal pathogen, ${ }^{19}$ or bacterial suppression of antibiotic peptides synthesis in Drosophila. ${ }^{20}$ In particular, both a direct and an indirect immunosuppression of the melanization response has been shown in Aedes aegypti mosquitoes infected by $P$. gallinaceum..$^{21,22}$ However, despite the indication that the expression of one $A$. gambiae immune gene, $N O$ synthase (NOS), is repressed after $P$. falciparum infection, ${ }^{3}$ it is unknown whether $P$. falciparum can suppress the melanization immune response of Anopheles mosquitoes. ${ }^{23}$

To evaluate the possibility of suppression of the melanization response by $P$. falciparum in $A$. gambiae, we compared the melanization ability of three classes of mosquitoes: those that became infected after an infectious blood meal, those that did not become infected (or cleared the parasite) after the blood meal, and those that were fed on an uninfected blood meal. Like previous studies, ${ }^{21,22,24}$ we quantified the 
melanization response by inoculating negatively charged Sephadex beads into the insect's hemolymph. The infection status of challenged mosquitoes was determined at 48 hours post-blood meal (pbm) by detection of Pfs 25 mRNA. ${ }^{25,26}$ Bead melanization was measured between 24 and 48 hours pbm during the transition between $P$. falciparum's ookinetes and oocysts, the developmental period of the parasite that is most susceptible to the melanization response, ${ }^{8}$ and when this response is most likely to be suppressed by $P$. gallinaceum. .1,22 $^{2}$

\section{MATERIALS AND METHODS}

General design. The study consisted of two experimental blocks (i.e., two repetitions of the same experiment). In each of the two blocks, we used two $P$. falciparum isolates (obtained from naturally infected gametocyte carriers) and one control blood sample (obtained from an uninfected volunteer) to feed mosquitoes from the same batch. We injected one CM-25 Sephadex bead into the thorax of each mosquito at 24 hours pbm and recovered it 24 hours later (i.e., 48 hours pbm) by dissecting the thorax. Because oocysts are too small at this stage to be detected by light microscopy, the corresponding abdomens (including the midgut) were stored individually in RNA Later (Ambion, Austin, TX) for further parasite detection by reverse transcription-polymerase chain reaction (RT-PCR). For each isolate or control blood sample, we used at least two replicate groups of mosquitoes.

Mosquitoes. The mosquitoes came from a colony of $A$. gambiae s.s. originating from Yaoundé that had previously been adapted to feed through Parafilm membrane. ${ }^{27}$ We recorded the mosquitoes' wing lengths, measured from the tip (excluding the fringe) to the distal end of the alula with a precision of $0.04 \mathrm{~mm}$, as an indication of body size. ${ }^{28}$ Where both wings could be measured, the mean of the two lengths was used.

Gametocyte carriers. Plasmodium falciparum carriers were recruited among 5- to 11-year-old children from primary schools in Mfou, a small town located $25 \mathrm{~km}$ east of Yaoundé, Cameroon. Thick blood smears were made from finger-prick blood samples, stained with $10 \%$ Giemsa for 20 minutes, and examined microscopically for $P$. falciparum gametocytes. Asymptomatic gametocyte-positive children were selected for the study after their parents or guardians had signed an informed consent form. Gametocyte carriers with malaria species other than $P$. falciparum were excluded from the study. All children with asexual parasitemia $(>1,000$ parasites $/ \mu \mathrm{L})$ were treated with an artemisinin-amodiaquine combination on the day after the screening, according to national guidelines for the treatment of simple malaria cases. The protocol obtained approval of the National Ethics Committee of Cameroon.

Experimental infections. Gametocyte carriers that had been identified the previous day were brought to the laboratory. A sample of $5 \mathrm{~mL}$ of venous blood was collected from each gametocyte carrier in a heparinized tube. Gametocyte density per 500 leukocytes was assessed just before blood withdrawal on a blood smear (as described above) and was converted to numbers of parasites per microliter by assuming a standard leukocyte count of $8,000 / \mu \mathrm{L}$. To standardize the blood meal, we centrifuged the blood at $37^{\circ} \mathrm{C}$ for 3 minutes at $2,000 \mathrm{~g}$ and replaced the autologous serum with non-immune
$\mathrm{AB}$ serum (the same $\mathrm{AB}$ serum was used for all infections), adjusting the hematocrit to $50 \%$. A sample of venous blood from an uninfected human volunteer was treated in the same manner and used as a control blood meal. Three-day-old female mosquitoes deprived of sugar for 5 hours before blood feeding were allowed to feed on the mixture for 30 minutes through standard membrane feeders. ${ }^{27}$ In each experimental block, replicate groups of 15-35 females maintained in paper cups covered with netting were simultaneously placed under membrane feeders containing either infected blood or uninfected blood. At least two different feeders were used for each infected or control blood sample, so that we could control for a potential effect of the feeder (included in the effect of replicate). After the blood meal, unfed and partially fed mosquitoes were discarded, and fully fed mosquitoes were kept in the insectary with permanent access to a $10 \%$ sucrose solution.

Melanization assays. CM-25 Sephadex beads (SigmaAldrich, Steinheim, Germany) range from 40 to $120 \mu \mathrm{m}$ in diameter; the smallest ones were selected by visual inspection for inoculation. Beads were rehydrated in saline solution containing $1.3 \mathrm{mmol} / \mathrm{L} \mathrm{NaCl}, 0.5 \mathrm{mmol} / \mathrm{L} \mathrm{KCl}$, and $0.2 \mathrm{mmol} / \mathrm{L}$ $\mathrm{CaCl}_{2}$ (pH 6.8) and stained with $0.001 \%$ methyl green to help in visualization. ${ }^{24} \mathrm{We}$ immobilized mosquitoes briefly on ice and injected one bead per mosquito into the thorax with $<0.1$ $\mu \mathrm{L}$ of saline solution into the hemolymph, using a heat-pulled capillary needle. ${ }^{24}$ After 24 hours, mosquitoes that were able to fly were dissected in saline solution with $0.01 \%$ methyl green. Beads were recovered, and melanization was scored according to three broad categories: weak melanization (class 0 ), intermediate melanization (class 1 ), and strong melanization (class 2). Figure 1A shows typical pictures of the three melanization classes.

Plasmodium falciparum detection by RT-PCR. The presence of live parasites was detected by RT-PCR of Pfs 25 mRNA in individual abdomens dissected at 48 hours pbm, after bead removal of the corresponding thorax. The $P f_{s} 25$ gene encodes a major surface protein of zygotes, ookinetes, and oocysts. ${ }^{25,26}$

Total RNA was extracted from each abdomen using the Tri Reagent kit (M.R.C., Ontario, Canada) according to the manufacturer's instructions. Extracted RNA was treated with the Turbo DNA-free kit (Ambion) and resuspended in $10 \mu \mathrm{L}$ of water with $1 \mu \mathrm{L}$ of RNasin (Promega, Madison, WI). RNA was reverse transcribed, and RT products were amplified using the Access RT-PCR System kit (Promega) according to the manufacturer's instructions on $1 \mu \mathrm{L}$ of the RNA extract in a final volume of $25 \mu \mathrm{L}$. Reverse transcription was performed for 45 minutes at $45^{\circ} \mathrm{C}$ followed by 2 minutes at $94^{\circ} \mathrm{C}$. Amplification conditions were five touchdown cycles (30 seconds at $94^{\circ} \mathrm{C}, 1$ minute at $55-50^{\circ} \mathrm{C}, 2$ minutes at $68^{\circ} \mathrm{C}$ ), followed by 20 cycles $\left(30\right.$ seconds at $94^{\circ} \mathrm{C}, 1$ minute at $50^{\circ} \mathrm{C}, 2$ minutes at $\left.68^{\circ} \mathrm{C}\right)$, and a final 7 -minute elongation step $\left(68^{\circ} \mathrm{C}\right)$. Absence of contaminating genomic DNA was systematically checked by a control without RT. A nested PCR was performed on 1 $\mu \mathrm{L}$ of a $1 / 100$ dilution of the RT-PCR products in a final volume of $25 \mu \mathrm{L}$. Amplification conditions were 2 minutes at $94^{\circ} \mathrm{C}$, followed by five touchdown cycles $\left(30\right.$ seconds at $94^{\circ} \mathrm{C}$, 30 seconds at $55-50^{\circ} \mathrm{C}, 30$ seconds at $72^{\circ} \mathrm{C}$ ) followed by 35 cycles $\left(30\right.$ seconds at $94^{\circ} \mathrm{C}, 30$ seconds at $50^{\circ} \mathrm{C}, 30$ seconds at $\left.72^{\circ} \mathrm{C}\right)$, and a final 7 -minute elongation step $\left(72^{\circ} \mathrm{C}\right)$. The specific primers used in the two PCR series have been described 
elsewhere. $^{3}$ Nested PCR products were analyzed on $1.2 \%$ agarose gels in Tris-borate buffer. Total RNA from cultured $P$. falciparum gametocytes was used as a positive control. A minimum detection threshold of $0.1 \mathrm{pg}$ of total $P$. falciparum RNA per $100 \mathrm{ng}$ of mosquito RNA was determined in a pilot experiment using dilutions of gametocyte RNA into mosquito RNA.

Data analysis. Because the use of Sephadex beads has the disadvantage that some mosquitoes die of the side effects of inoculation, we first controlled for a potential bias caused by post-inoculation mortality. We analyzed the arsinetransformed mortality rates among replicates as a function of the experimental treatment (control blood versus challenge by $P$. falciparum), including the experimental block as a potential random confounder.

We analyzed the data in two steps. First, we determined whether the melanization response of challenged mosquitoes (i.e., those that fed on infected blood) was associated with infection success at 48 hours pbm. The level of bead melanization (scored as three categories) was analyzed with an ordinal logistic analysis as a function of the mosquito's infection status (infected or uninfected). Wing length (an indication of body size), the experimental block, the parasite isolate, and the replicate were included as potential confounders. As different isolates were used in each block, isolate was nested within block. Replicate was nested within block and isolate; block, isolate, and replicate were considered as random factors. Second, we compared the melanization response between mosquitoes challenged with $P$. falciparum and control mosquitoes fed on an uninfected blood meal. Because the first step of the analysis had revealed differences in the melanization level of infected and uninfected mosquitoes, we compared these two groups to control mosquitoes separately. For each group (infected or uninfected), the level of bead melanization (scored into three categories) was analyzed with an ordinal logistic analysis as a function of the blood-meal type (infectious or control). The wing length, the experimental block, and the replicate were included as potential confounders. Replicate was nested within block; block and replicate were considered as random factors. We did not include the isolate as a confounder because the first analysis showed that there was no difference between isolates.

All analyses were performed with the software JMP version 5.0 (http://www.jmpdiscovery.com).

\section{RESULTS}

Overall, we measured the melanization response of 153 mosquitoes, 104 of which had been fed with infected blood and 49 with control blood. The four blood samples corresponding to the four $P$. falciparum isolates had similar gametocyte densities $(\sim 16$ gametocytes/ $\mu \mathrm{L})$. $P f s 25$ mRNA was detected in $63(60.6 \%)$ of challenged mosquitoes 48 hours pbm. Infection rates by isolate were $74.1 \%$ and $79.4 \%$ in the first experimental block and $32 \%$ and $30.8 \%$ in the second block, respectively. Post-inoculation mortality of challenged mosquitoes was $28.8 \%$ and $18.2 \%$ in the first and the second block, respectively, and $30.3 \%$ and $33.6 \%$ in control mosquitoes. There was no statistical effect of the block $\left(F_{1,8}=0.3, P\right.$ $=0.599)$, the challenge $\left(F_{1,8}=1.5, P=0.255\right)$, or their interaction $\left(F_{1,8}=0.88, P=0.376\right)$ on mortality rates.

On average, melanization was stronger in the second experimental block (Figure 1), which may be because of the difference in body size of the two corresponding batches of mosquitoes. Indeed, the mean wing length was $3.09 \pm 0.020$ (SE) $\mathrm{mm}$ in the first block and $3.39 \pm 0.015 \mathrm{~mm}$ in the second block, and melanization was overall positively associated to the wing length in a one-way logistic analysis $\left(\chi^{2}=6.04, P=\right.$ $0.014)$.

Among females fed on infectious blood, the mosquitoes with live parasites at 48 hours pbm had a significantly stronger melanization response than those where no parasite was detected (Table 1). Although $40 \%$ and $93.8 \%$, respectively, of
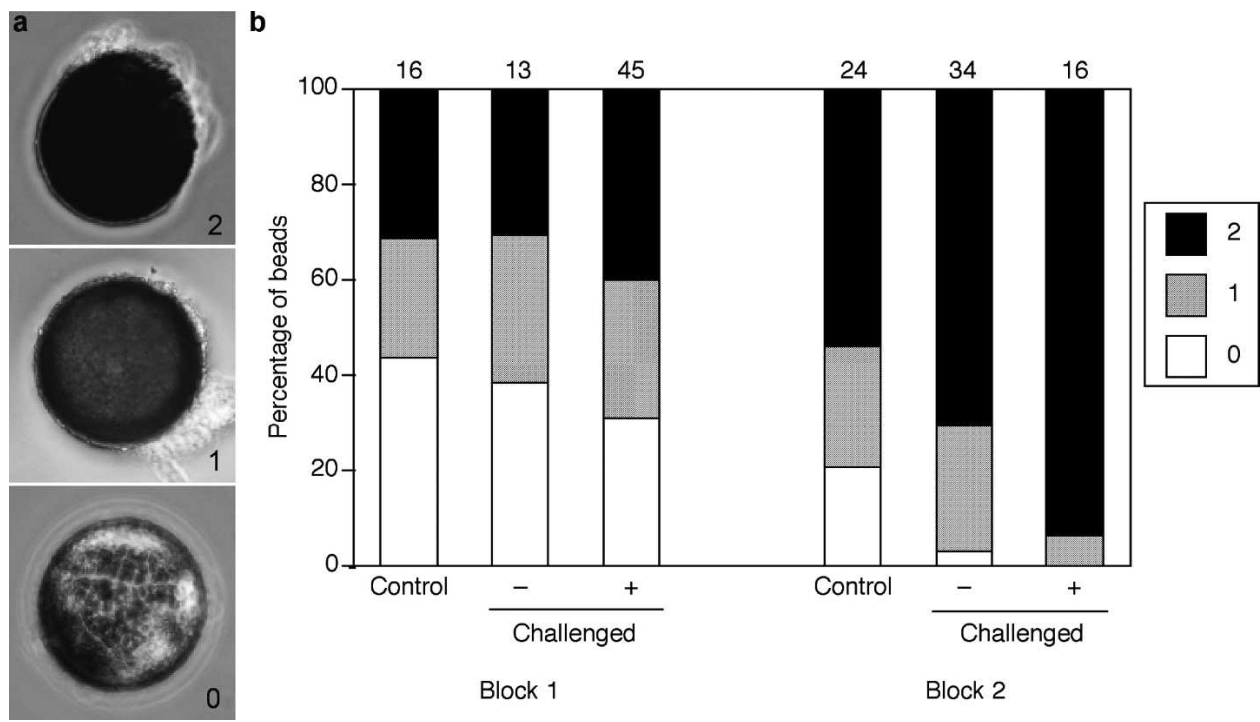

Figure 1. Melanization response of mosquitoes infected by $P$. falciparum. (A) Typical examples of the three broad classes of bead melanization, ranging from strong melanization (2) on the top to weak melanization (0) on the bottom. (B) Proportion of mosquitoes in the three melanization classes for mosquitoes challenged by $P$. falciparum or fed on uninfected (control) blood. Challenged mosquitoes are divided into individuals that were found to be infected $(+)$ or uninfected $(-) 48$ hours pbm. Numbers of mosquitoes are given above each bar. 
TABLE 1

Test statistics for the comparison of the melanization response of mosquitoes determined to be either infected or uninfected by $P$. falciparum at 48 hours after an infectious blood meal

\begin{tabular}{lccr}
\hline \multicolumn{1}{c}{ Source } & df & Likelihood-ratio $\chi^{2}$ & \multicolumn{1}{c}{$P$} \\
\hline Wing length & 1 & 0.797 & 0.372 \\
Experimental block & 1 & 23.02 & $<0.001$ \\
Infection status & 1 & 4.11 & 0.043 \\
Infection $\times$ block & 1 & 1.68 & 0.195 \\
Isolate (within block) & 2 & 0.360 & 0.835 \\
Replicate (within block and isolate) & 4 & 7.91 & 0.095 \\
\hline
\end{tabular}

the infected mosquitoes strongly melanized the bead in the first and the second block, only $30.8 \%$ and $70.6 \%$ did so in the uninfected mosquitoes (Figure 1).

Among control females (fed on uninfected blood), 31.3\% and $54.2 \%$, respectively, of mosquitoes strongly melanized the bead in the first and in the second block. There was no statistically significant difference between the level of melanization of challenged but uninfected mosquitoes and that of control mosquitoes (Table 2). In contrast, the melanization level was highly significantly higher in infected mosquitoes compared with the controls (Table 2). This difference was stronger in the second block, as revealed by the significant effect of the interaction between block and blood-meal type (Table 2).

\section{DISCUSSION}

We found that $A$. gambiae mosquitoes were able to melanize inoculated Sephadex beads when infected by $P$. falciparum and that the melanization response of mosquitoes harboring live parasites was slightly stronger than that of mosquitoes exposed to the parasite but in which the infection had failed. This result contrasts previous studies showing strong immuno-suppression of the melanization response by $P$. gallinaceum in experimentally infected Ae. aegypti mosquitoes, ${ }^{21,22}$ indicating that the two species of malaria parasites rely on different strategies to avoid the mosquito's immune response. To explain this difference, it is important to consider that we used a co-evolved system of $A$. gambiae and $P$. falciparum, in contrast to the model system of Ae. aegypti and $P$. gallinaceum. The influence of co-evolution has previously been pointed out by the observation that a refractory strain of A. gambiae that efficiently melanizes New World and Asian strains of $P$. falciparum fails to melanize African strains of $P$. falciparum. ${ }^{8}$ Because the geographic range of $A$. gambiae is restricted to sub-Saharan Africa, this observation suggests that sympatric malaria parasites have evolved local adaptations to overcome the melanization response, as has been observed in other host-parasite systems. ${ }^{29}$ Thus, it is possible that in the co-evolved system of A. gambiae and P. falciparum, the mosquitoes have evolved a mechanism to avoid having their melanization response suppressed, leading to a counter-adaptation of the parasites based on a different strategy. Alternatively, theoretical considerations of a coevolutionary system have suggested that, in an area of intense transmission such as the one studied here, co-evolutionary pressure might lead to a situation where mosquitoes have not evolved immune resistance against malaria and where, therefore, the parasites have not evolved immuno-suppressive mechanisms. $^{30}$

That the melanization response was slightly stronger in infected than in uninfected mosquitoes could result from a trade-off between bead melanization and parasite resistance, whereby mosquitoes able to clear early stages of the parasite would have a weaker melanization response against beads and vice-versa. Such a trade-off could be caused by a negative genetic correlation between different components of the immune system. ${ }^{31}$ This idea is supported by the observation that alleles responsible for $P$. falciparum melanization are associated to high parasite numbers in wild pedigrees of $A$. gambiae ${ }^{32}$ but contrasts a previous study showing that the genetic correlation between two arms of the immune systemantibacterial defense and melanization-is positive rather than negative in a natural population of A. gambiae. ${ }^{33} \mathrm{How}-$ ever, the existence of a trade-off between parasite resistance and bead melanization would imply in our study that the mosquitoes that cleared the parasite would have, on average, a weaker melanization response than mosquitoes fed on uninfected blood. This was not the case; rather, the melanization response was similar in the two categories of mosquitoes (Table 2). It is thus more likely that the melanization response had been triggered after midgut invasion by the early stages of malaria parasites. This could simply result from the cell damage caused by ookinetes during their migration through the midgut epithelium, ${ }^{34}$ because melanization is also intimately tied to wound healing, ${ }^{7}$ or from an immunologic priming $^{35}$ following the previous up regulation of immune components caused by the detection of invading malaria parasites. ${ }^{3}$

The lack of immuno-suppressive ability of the melanization response in a natural population of $P$. falciparum suggests that the parasite survives this response by evading it. Note that this might not hold for other immune responses that can affect Plasmodium development. For example, the expression of the NOS gene of $A$. gambiae is repressed after $P$. falciparum infection, ${ }^{3}$ suggesting that the parasite might reduce

TABLE 2

Test statistics for the comparison of the melanization response between challenged mosquitoes determined to be uninfected or infected after an infectious blood meal and control mosquitoes fed on uninfected blood

\begin{tabular}{|c|c|c|c|c|c|}
\hline \multirow[b]{2}{*}{ Source } & \multirow[b]{2}{*}{ df } & \multicolumn{2}{|c|}{ Control vs uninfected } & \multicolumn{2}{|c|}{ Control vs infected } \\
\hline & & Likelihood ratio $\chi^{2}$ & $P$ & Likelihood ratio $\chi^{2}$ & $P$ \\
\hline Wing length & 1 & 0.455 & 0.500 & 0.191 & 0.662 \\
\hline Experimental block & 1 & 7.15 & 0.008 & 14.5 & $<0.001$ \\
\hline Blood meal type & 1 & 1.07 & 0.302 & 8.70 & 0.003 \\
\hline Blood $\times$ block & 1 & 1.28 & 0.258 & 5.91 & 0.015 \\
\hline Replicate (within block and blood) & 8 & 12.8 & 0.118 & 9.78 & 0.281 \\
\hline
\end{tabular}


the production of NO, a molecule known to limit Plasmodium development in the mosquito. ${ }^{36}$ Immune evasion mechanisms by parasitic protozoa in their vertebrate hosts include antigenic variation, shedding of surface proteins, antigenic mimicry, and intracellular hiding, ${ }^{37}$ but such mechanisms in the vector remain unknown. Candidate molecules enabling the evasion of the melanization response by the parasite include mosquito immune proteins acting as protective agonists. For instance, two C-type lectins, CTL4 and CTLMA2, prevent the melanization of $P$. berghei by a susceptible line of $A$. gambiae. ${ }^{10}$ Moreover, gene silencing of CTL4 or CTLMA2 does not affect the melanization of Sephadex beads in $A$. gambiae, supporting the idea that the parasite might specifically use these molecules to evade the immune response. ${ }^{38}$

In conclusion, this study showed that experimental infection of $A$. gambiae by $P$. falciparum is not associated with an immuno-suppression but rather an enhanced melanization response. This contrasts earlier laboratory-based studies with $P$. gallinaceum, where the parasite suppressed the melanization response of $A$ e. aegypti. ${ }^{21,22}$ Thus, together with another study, ${ }^{3}$ our finding confirms the need to validate the observations made in laboratory models of mosquito-malaria interactions in field transmission systems. ${ }^{23,39}$ It also stresses the importance of addressing the strategies used by malaria parasites to overcome mosquito defense mechanisms.

Received September 13, 2006. Accepted for publication November 18, 2006.

Acknowledgments: We thank the inhabitants of Mfou for cooperation and Engelbert Manga, Constance Efemba, and Emmanuel Bozewan for kind support during the fieldwork in Mfou. The authors thank Isaac Tchikangwa for rearing mosquitoes, Rose Nyambam and Sylvie Kemleu-Zebaze for assistance in the screening of gametocyte carriers, Kristin Michel for help during the fieldwork and for the images of beads, Isabelle Thiéry for providing cultured $P$. falciparum gametocytes, and an anonymous reviewer for helpful comments on an earlier version of the manuscript.

Financial support: This study was supported by the VIHPAL Program of the French Ministry of Research.

Authors' addresses: Louis Lambrechts and Jacob C. Koella, Laboratoire de Parasitologie Evolutive, Unité Mixte de Recherche 7103, Centre National de la Recherche Scientifique-Université Pierre et Marie Curie, Case Courrier 237, 7 quai St Bernard, 75252 Paris Cedex 05, France, Telephone: 33144275204, Fax: 33144273516. Present address of Louis Lambrechts, Department of Entomology, University of California, One Shields Ave., Davis, CA 95616, Telephone: 5307520565, Fax: 5307521537. Present address of Jacob C. Koella, Division of Biology, Imperial College London, Silwood Park Campus, Ascot SL5 7PY, UK, Telephone: 442075942254. Louis Lambrechts, Jean-Claude Jacques, and Catherine Bourgouin, Centre de Production et d'Infection des Anophèles, Institut Pasteur, 28 rue du Dr Roux, 75724 Paris Cedex 15, France, Telephone: 33144389577, Fax: 33140613089. Isabelle Morlais, Parfait H. Awono-Ambene, and Frédéric Simard, Laboratoire de Recherche sur le Paludisme, Institut de Recherche pour le Développement-Organisation de Coordination pour la Lutte contre les Endémies en Afrique Centrale, BP 288, Yaoundé, Cameroon, Telephone: 2372232232/9932111, Fax: 2372230061/2201854. Isabelle Morlais, Anna Cohuet, and Frédéric Simard, Unité de Caractérisation et Contrôle des Populations de Vecteurs, Institut de Recherche pour le Développement, 911 Avenue Agropolis, BP 64501, 34394 Montpellier Cedex 5, France, Telephone: 33467041924, Fax: 33467542044. Catherine Bourgouin, Unité de Biologie et Génétique du Paludisme, Institut Pasteur, 28 rue du Dr Roux, 75724 Paris Cedex 15, France, Telephone: 33145688224, Fax: 33140613089.

Reprints requests: Louis Lambrechts, Department of Entomology, University of California, One Shields Ave., Davis, CA 95616. E-mail: llambrechts@ucdavis.edu.

\section{REFERENCES}

1. Christophides GK, 2005. Transgenic mosquitoes and malaria transmission. Cell Microbiol 7: 325-333.

2. Richman A, Kafatos FC, 1996. Immunity to eukaryotic parasites in vector insects. Curr Opin Immunol 8: 14-19.

3. Tahar R, Boudin C, Thiéry I, Bourgouin C, 2002. Immune response of Anopheles gambiae to the early sporogonic stages of the human malaria parasite Plasmodium falciparum. EMBO J 21: 6673-6680.

4. Michel K, Kafatos FC, 2005. Mosquito immunity against Plasmodium. Insect Biochem Mol Biol 35: 677-689.

5. Gouagna LC, Mulder B, Noubissi E, Tchuinkam T, Verhave JP, Boudin C, 1998. The early sporogonic cycle of Plasmodium falciparum in laboratory-infected Anopheles gambiae: An estimation of parasite efficacy. Trop Med Int Health 3: 21-28.

6. Vaughan JA, Noden BH, Beier JC, 1992. Population dynamics of Plasmodium falciparum sporogony in laboratory-infected Anopheles gambiae. J Parasitol 78: 716-724.

7. Christensen BM, Li J, Chen CC, Nappi AJ, 2005. Melanization immune responses in mosquito vectors. Trends Parasitol 21: 192-199.

8. Collins FH, Sakai RK, Vernick KD, Paskewitz S, Seeley DC, Miller LH, Collins WE, Campbell CC, Gwadz RW, 1986. Genetic selection of a Plasmodium-refractory strain of the malaria vector Anopheles gambiae. Science 234: 607-610.

9. Paskewitz SM, Brown MR, Lea AO, Collins FH, 1988. Ultrastructure of the encapsulation of Plasmodium cynomolgi (B strain) on the midgut of a refractory strain of Anopheles gambiae. J Parasitol 74: 432-439.

10. Osta MA, Christophides GK, Kafatos FC, 2004. Effects of mosquito genes on Plasmodium development. Science 303: 20302032.

11. Michel K, Budd A, Pinto S, Gibson TJ, Kafatos FC, 2005. Anopheles gambiae SRPN2 facilitates midgut invasion by the malaria parasite Plasmodium berghei. EMBO Rep 6: 891-897.

12. Volz J, Osta MA, Kafatos FC, Muller HM, 2005. The roles of two clip domain serine proteases in innate immune responses of the malaria vector Anopheles gambiae. J Biol Chem 280: 40161-40168.

13. Abraham EG, Pinto SB, Ghosh A, Vanlandingham DL, Budd A, Higgs S, Kafatos FC, Jacobs-Lorena M, Michel K, 2005. An immune-responsive serpin, SRPN6, mediates mosquito defense against malaria parasites. Proc Natl Acad Sci USA 102: 16327-16332.

14. Schwartz A, Koella JC, 2002. Melanization of Plasmodium falciparum and C-25 sephadex beads by field-caught Anopheles gambiae (Diptera: Culicidae) from southern Tanzania. J Med Entomol 39: 84-88.

15. Adini A, Warburg A, 1999. Interaction of Plasmodium gallinaceum ookinetes and oocysts with extracellular matrix proteins. Parasitology 119: 331-336.

16. Li X, Webb BA, 1994. Apparent functional role for a cysteinerich polydnavirus protein in suppression of the insect cellular immune response. J Virol 68: 7482-7489.

17. Rosqvist R, Forsberg A, Rimpilainen M, Bergman T, Wolf-Watz H, 1990. The cytotoxic protein YopE of Yersinia obstructs the primary host defence. Mol Microbiol 4: 657-667.

18. Rosqvist R, Forsberg A, Wolf-Watz H, 1991. Intracellular targeting of the Yersinia YopE cytotoxin in mammalian cells induces actin microfilament disruption. Infect Immun 59: 4562-4569.

19. Bouarab K, Melton R, Peart J, Baulcombe D, Osbourn A, 2002. A saponin-detoxifying enzyme mediates suppression of plant defences. Nature 418: 889-892.

20. Lindmark H, Johansson KC, Stoven S, Hultmark D, Engstrom Y, Soderhall K, 2001. Enteric bacteria counteract lipopolysaccharide induction of antimicrobial peptide genes. J Immunol 167: 6920-6923.

21. Boëte C, Paul RE, Koella JC, 2002. Reduced efficacy of the immune melanization response in mosquitoes infected by malaria parasites. Parasitology 125: 93-98.

22. Boëte C, Paul RE, Koella JC, 2004. Direct and indirect immunosuppression by a malaria parasite in its mosquito vector. Proc R Soc Lond B Biol Sci 271: 1611-1615.

23. Boëte $C, 2005$. Malaria parasites in mosquitoes: Laboratory mod- 
els, evolutionary temptation and the real world. Trends Parasitol 21: 445-447.

24. Paskewitz S, Riehle MA, 1994. Response of Plasmodium refractory and susceptible strains of Anopheles gambiae to inoculated Sephadex beads. Dev Comp Immunol 18: 369-375.

25. Lensen AH, Van Gemert GJ, Bolmer MG, Meis JF, Kaslow D, Meuwissen JH, Ponnudurai T, 1992. Transmission blocking antibody of the Plasmodium falciparum zygote/ookinete surface protein Pfs 25 also influences sporozoite development. Parasite Immunol 14: 471-479.

26. Vermeulen AN, van Deursen J, Brakenhoff RH, Lensen TH, Ponnudurai T, Meuwissen JH, 1986. Characterization of Plasmodium falciparum sexual stage antigens and their biosynthesis in synchronised gametocyte cultures. Mol Biochem Parasitol 20: $155-163$.

27. Tchuinkam T, Mulder B, Dechering K, Stoffels H, Verhave JP, Cot M, Carnevale P, Meuwissen JH, Robert V, 1993. Experimental infections of Anopheles gambiae with Plasmodium falciparum of naturally infected gametocyte carriers in Cameroon: Factors influencing the infectivity to mosquitoes. Trop Med Parasitol 44: 271-276.

28. Koella JC, Lyimo EO, 1996. Variability in the relationship between weight and wing length of Anopheles gambiae (Diptera: Culicidae). J Med Entomol 33: 261-264.

29. Rigaud T, Moret Y, 2003. Differential phenoloxidase activity between native and invasive gammarids infected by local acanthocephalans: Differential immunosuppression? Parasitology 127: 571-577.

30. Koella JC, Boëte C, 2003. A model for the coevolution of immunity and immune evasion in vector-borne diseases with implications for the epidemiology of malaria. Am Nat 161: 698-707.

31. Cotter SC, Kruuk LE, Wilson K, 2004. Costs of resistance: Ge- netic correlations and potential trade-offs in an insect immune system. J Evol Biol 17: 421-429.

32. Riehle MM, Markianos K, Niare O, Xu J, Li J, Toure AM, Podiougou B, Oduol F, Diawara S, Diallo M, Coulibaly B, Ouatara A, Kruglyak L, Traore SF, Vernick KD, 2006. Natural malaria infection in Anopheles gambiae is regulated by a single genomic control region. Science 312: 577-579.

33. Lambrechts L, Vulule JM, Koella JC, 2004. Genetic correlation between melanization and antibacterial immune responses in a natural population of the malaria vector Anopheles gambiae. Evolution Int J Org Evolution 58: 2377-2381.

34. Baton LA, Ranford-Cartwright LC, 2005. How do malaria ookinetes cross the mosquito midgut wall? Trends Parasitol 21: 22-28.

35. Moret Y, Siva-Jothy MT, 2003. Adaptive innate immunity? Responsive-mode prophylaxis in the mealworm beetle, Tenebrio molitor. Proc Biol Sci 270: 2475-2480.

36. Luckhart S, Vodovotz Y, Cui L, Rosenberg R, 1998. The mosquito Anopheles stephensi limits malaria parasite development with inducible synthesis of nitric oxide. Proc Natl Acad Sci USA 95: 5700-5705.

37. Zambrano-Villa S, Rosales-Borjas D, Carrero JC, Ortiz-Ortiz L, 2002. How protozoan parasites evade the immune response. Trends Parasitol 18: 272-278.

38. Warr E, Lambrechts L, Koella JC, Bourgouin C, Dimopoulos G, 2006. Anopheles gambiae immune responses to Sephadex beads: Involvement of anti-Plasmodium factors in regulating melanization. Insect Biochem Mol Biol 36: 769-778.

39. Aguilar R, Dong Y, Warr E, Dimopoulos G, 2005. Anopheles infection responses; laboratory models versus field malaria transmission systems. Acta Trop 95: 285-291. 